\title{
Vedolizumab Induces Endoscopic and Histologic Remission in Patients With Crohn's Disease
}

\author{
a14 Mark Löwenberg, ${ }^{1}$ Severine Vermeire, ${ }^{2}$ Nahid Mostafavi, ${ }^{3}$ Frank Hoentjen, ${ }^{4}$
} Denis Franchimont, ${ }^{5}$ Peter Bossuyt, ${ }^{6}$ Pieter Hindryckx, ${ }^{7}$ Theo Rispens, ${ }^{8}$ Annick de Vries, ${ }^{8}$ C. Janneke van der Woude, ${ }^{9}$ Sophie Berends,,${ }^{1,10}$ Carmen A. Ambarus, ${ }^{11}$ Ron Mathot,,${ }^{1,10}$ Esme Clasquin, ${ }^{1}$ Filip Baert, ${ }^{12}$ and Geert D'Haens ${ }^{1}$

\begin{abstract}
${ }^{1}$ Amsterdam UMC, University of Amsterdam, Department of Gastroenterology and Hepatology, Amsterdam, The Netherlands; ${ }^{2}$ Division of Gastroenterology and Hepatology, University Hospitals Leuven, Leuven, Belgium; ${ }^{3}$ Amsterdam UMC, University of Amsterdam, Biostatistics Unit of Department of Gastroenterology and Hepatology, Amsterdam, The Netherlands; ${ }^{4}$ Department of Gastroenterology and Hepatology, Radboud University Medical Center, Nijmegen, The Netherlands; ${ }^{5}$ Department of Gastroenterology, Erasme Hospital - Université Libre de Bruxelles 808, Brussels, Belgium; ${ }^{6}$ Imelda GI Clinical Research Center, Imelda General Hospital, Bonheiden, Belgium; ' University Hospital of Ghent, Department of Gastroenterology, Ghent, Belgium; ${ }^{8}$ Biologics Lab, Sanquin Diagnostic Services, Amsterdam, The Netherlands; ${ }^{9}$ Erasmus MC, Department of Gastroenterology, Rotterdam, The Netherlands; ${ }^{10}$ Amsterdam UMC, University of Amsterdam, Hospital Pharmacy, Amsterdam, The Netherlands; ${ }^{11}$ Amsterdam UMC, University of Amsterdam, Department of Pathology, Amsterdam, The Netherlands; and ${ }^{12}$ AZ Delta, Division of Gastroenterology, Roeselare, Belgium
\end{abstract}

BACKGROUND \& AIMS: We evaluated the ability of vedolizumab to induce endoscopic and histologic remission in patients with Crohn's disease (CD). METHODS: We performed a prospective study of 110 patients with active CD, based on CD activity index (CDAI) scores $>220$ and mucosal ulcerations, who received open-label vedolizumab (300 $\mathrm{mg}$ ) infusions at weeks 0,2 , and 6 , and every 8 weeks thereafter through week 52 at tertiary centers in Europe. Patients received an additional infusion at week 10 if their CDAI score had not decreased by 70 points. Patients underwent ileocolonoscopy with collection of biopsies at baseline and weeks 26 and 52; a local and central reader determined simple endoscopic index for CD (SES-CD) scores. Histologic features were assessed by a blinded pathologist at week 26. Serum concentrations of vedolizumab were measured at serial time points. The primary outcome was endoscopic and histologic remission in patients with active CD treated with vedolizumab for 52 weeks. RESULTS: At weeks 26 and 52,36 patients (29\%) and 34 patients (31\%), respectively, were in corticosteroid-free clinical remission (CDAI score $<150$ ), respectively. Based on intent-to-treat analysis, endoscopic remission (SES-CD score $<4$ ) was achieved by 36 patients (33\%) and 40 patients (36\%) at weeks 26 and 52 . Endoscopic responses (decrease in SES-CD score $\geq 50 \%$ ) occurred in 44 patients (40\%) at week 26 and 5 patients $(45 \%)$ at week 52. Serum concentrations of vedolizumab were higher at weeks 2, 10, and 22 in patients with lower SES-CD scores. Histologic remission at week 26 was observed in $43(64 \%)$ of 67 patients based on Geboes Score and 37 (66\%) of 56 patients based on Robarts Histopathology Index scores in analyses of paired biopsies with inflammation at baseline. Serum concentrations of vedolizumab above $10 \mathrm{mg} / \mathrm{L}$ at week 22 were associated with endoscopic remission at week 26. CONCLUSIONS: In a prospective trial, we found that approximately one-third of patients with $\mathrm{CD}$ achieve endoscopic remission after 52 weeks of treatment with vedolizumab and two-thirds achieve histologic remission at week 26. Higher serum concentrations of vedolizumab were associated with better outcomes. EUDRACT no: 2014-005376-29.
Keywords: LOVE-CD Trial; IBD; Biologic; Anti-integrin $\alpha_{4} ; \beta_{7}$.

Crohn's disease (CD) is a chronic disabling disease that can affect the entire gastrointestinal tract. $^{1}$ Symptoms commonly include abdominal pain, diarrhea, weight loss, and fatigue. Vedolizumab (VDZ) is a gutselective humanized monoclonal antibody that binds to the $\alpha 4 \beta 7$ integrin, thereby inhibiting leucocyte vascular adhesion and migration into the gastrointestinal mucosa. VDZ is approved for the treatment of moderate to severe CD, based on results of the GEMINI 2 and 3 phase 3 randomized controlled trials. ${ }^{2,3}$ Although the efficacy and safety of VDZ induction and maintenance therapy in $\mathrm{CD}$ has been confirmed in real-life cohorts, only limited data are available on endoscopic and histological remission. ${ }^{4-8}$

Endoscopic remission is an important treatment goal in CD that is associated with improved clinical outcomes, including reduced hospitalization and surgery rates. ${ }^{9,10}$ Moreover, endoscopic response is recommended as a coprimary endpoint in clinical trials by regulatory agencies. $^{11}$ Nonetheless, only a few studies have investigated endoscopic remission with VDZ in CD and these have reported on highly selected patients. Noman and colleagues ${ }^{4}$ studied 29 patients with CD who participated in the openlabel extension phase of the GEMINI 2 trial. Endoscopic

Abbreviations used in this paper: AVA, anti-vedolizumab antibodies; $C D$, Crohn's disease; CDAI, Crohn's Disease Activity Index; CI, confidence interval; CR, central reader; CRP, C-reactive protein; GS, Geboes Score; LOVE-CD, LOw countries VEdolizumab in CD; RHI, Robarts Histopathology Index; ROC, receiver operating characteristic; SES-CD, Simple Endoscopic Score for CD; SR, site reader; TNF, tumor necrosis factor; VDZ, vedolizumab. 


\section{WHAT YOU NEED TO KNOW}

\section{BACKGROUND AND CONTEXT}

Although the efficacy and safety of vedolizumab induction and maintenance therapy of Crohn's disease (CD) has been confirmed, few data are available on endoscopic and histologic remission.

\section{NEW FINDINGS}

Vedolizumab treatment induces endoscopic and histologic remission in patients with CD. Higher serum concentrations of vedolizumab are associated with endoscopic remission. Serum concentrations of vedolizumab above $10 \mathrm{mg} / \mathrm{L}$ at week 22 were associated with endoscopic remission at week 26 .

\section{LIMITATIONS}

This was an open-label study design. However, endoscopic and histologic outcomes were assessed blindly.

\section{IMPACT}

These findings can be used in prospective doseoptimization studies, to determine whether dose intensification of vedolizumab can further improve outcomes in patients with CD.

remission, defined as disappearance of ulcers seen at endoscopy, was observed in 29\% of patients receiving VDZ. In the largest experience, Dulai et $\mathrm{al}^{5}$ and the Victory consortium reported results from a cohort study of 212 patients with CD who received VDZ and showed a high 12-month cumulative endoscopic remission rate of $63 \%$ (defined as absence of ulcers and/or erosions) in a subset of patients. However in this study, endoscopy was performed according to clinical practice, in distinction to predefined intervals, and it was unclear how these patients were selected for inclusion in the analyses. Importantly, site investigators evaluated the endoscopies, in distinction to blinded central readers (CRs). This circumstance may have resulted in an overestimation of treatment efficacy.

In the past decade, multiple studies of tumor necrosis factor (TNF) antagonists have shown better clinical outcomes in patients with higher drug exposures. ${ }^{12-14}$ Likewise, a positive association between VDZ serum concentrations and clinical remission was reported in a post hoc analysis of the GEMINI 2 and 3 trials. ${ }^{15}$ However, exposure-efficacy relationships for endoscopic and histological outcomes, 2 objective measures of pharmacodynamic effects, have not been previously reported in VDZ-treated patients with CD.

The primary objective of the present study was to explore endoscopic and histological outcomes in patients with active CD treated with VDZ for 52 weeks. As a secondary objective, we evaluated pharmacokinetic/pharmacodynamic relationships for these endpoints (LOVE-CD: LOw countries VEdolizumab in CD; EUDRACT number: 2014-005376-29).

\section{Materials and Methods}

In LOVE-CD, adult patients with $C D$ ( $\geq 18$ years of age) were enrolled at 22 sites in Belgium and the Netherlands.
Participants had moderately to severely active CD with a CD activity index (CDAI) between 220 and 450 at screening and with objective evidence of ulcerations at baseline endoscopy. Patients who previously failed or were intolerant to TNF antagonist or those naïve to these agents were eligible. Following screening, patients received open-label intravenous treatment with $300 \mathrm{mg} \mathrm{VDZ}$ at weeks 0 , 2, and 6 (induction treatment), followed by maintenance treatment with $300 \mathrm{mg}$ VDZ every 8 weeks. An additional VDZ infusion was given at week 10 to patients who had no clinical response, defined as failure to achieve a $>70$-point CDAI decrement from baseline. Patients received treatment with VDZ for 1 year, which was the time point of the final assessment. All background CD medication was kept stable during the trial except for corticosteroids, which were tapered once clinical response was attained until complete withdrawal by week 26, if possible. Patients were considered in clinical remission only if their CDAI score was $<150$ with complete withdrawal of corticosteroids. All patients provided written informed consent. The study protocol was approved by the investigational review board at each study center. All authors had access to the study data and reviewed and approved the final manuscript.

\section{Procedures}

Assessments included physical examination, monitoring of laboratory data including C-reactive protein (CRP), CDAI scoring, and recording of adverse events at baseline and before every infusion. Serum was analyzed for VDZ concentrations and anti-VDZ antibodies (AVA) before every infusion in the first 26 weeks. Ileocolonoscopies were performed and video-recorded by experienced endoscopists (site readers [SRs]) at baseline and weeks 26 and 52. The Simple Endoscopic Score for Crohn's Disease (SES-CD), which evaluates size of ulcers, ulcerated surface, affected surface, and presence of stenosis in 4 segments of the colon and in the terminal ileum, was used for scoring. Total scores range from 0 to 56 points, with higher scores reflecting more severe disease. ${ }^{16}$ Patients who withdrew from the study before week 26 or week 52 had an exit endoscopy. Video recordings were also centrally reviewed by 4 expert CRs who were unaware of the study visit sequence or clinical information. The CR score was used for final analysis except when CR and SR scoring were discrepant, defined as follows. If the SR SES-CD was 0 to 3, a 1-point difference between SR and CR was considered a discrepancy. For SR SES-CD 4 to 7,8 to 15 , and $\geq 16$, a difference $>2$ points, $>3$ points, and $>4$ points between SR and CR was considered to represent discrepancy. ${ }^{17}$ Videos with discrepant reads were sent for adjudication by 2 other members of the CR team in an adjudication meeting, during which the video and the previous scores were evaluated. The SR or CR score closest to the adjudication read was used for further analysis.

Mucosal biopsies were obtained at baseline and at week 26 . Biopsies were collected from the edge of the most prominent ulcer in each segment (ie, ileum, ascending colon, transverse colon, descending colon and sigmoid, and rectum), or from the most severely affected area if no ulcers were present. If a segment was completely normal, 2 biopsies were taken at random per segment. Biopsies were stored in formalin and subsequently embedded in paraffin. The paraffin blocks were sectioned and stained with hematoxylin and eosin. Histological disease activity was scored blinded by an experienced
181

182

183

184

185

186

187

188

189

190

191

192

193

194

195

196

197

198

199

200

201

202

203

204

205

206

207

208

209

210

211

212

213

214

215

216

217

218

219

220

221

222

223

224

225

226

227

228

229

230

231

232

233

234

235

236

237

238

239

240 
pathologist using the Geboes Score (GS) ${ }^{18}$ and the Robarts Histopathology Index (RHI). ${ }^{19}$ The GS is a 7 -item scale (with 4 levels of severity for each item) that categorizes inflammation as grade 0 (architectural change only), grade 1 (chronic inflammation), grade 2 (2a, lamina propria eosinophils and $2 b$, lamina propria neutrophils), grade 3 (neutrophils in the epithelium), grade 4 (crypt destruction), or grade 5 (erosion or ulceration). The score ranges from 0 to 5.4 . The RHI is a 4-item index (with 4 levels for each item) that evaluates chronic inflammation, lamina propria neutrophils, neutrophils in the epithelium, and erosion or ulceration. Total score ranges from 0 to 33, where higher scores denote more severe inflammation. Associations between endoscopic remission (using SES-CD scores per segment; ie, terminal ileum, right colon, transverse colon, left colon, and rectum) and histological remission (using GS and RHI scores per segment) at week 26 were studied, as well as associations between clinical remission and both endoscopic and histological remission.

\section{VDZ Serum Concentrations and AVAs}

VDZ serum concentrations were measured at weeks $0,2,6$, $10,14,22,30,38,46$, and 52 using an immunoassay using rabbit AVAs to capture VDZ and rabbit anti-VDZ F(ab')2 fragments, as previously described (Sanquin Laboratories, Amsterdam, the Netherlands). ${ }^{20}$ The lower limit of quantification in serum is $100 \mathrm{ng} / \mathrm{mL}$; interassay precision and accuracy are $1 \%$ to $4 \%$ and $87 \%$ to $115 \%$, respectively. AVAs were also measured as previously described. ${ }^{21,22}$ The lower limit of detection was based on mean $>3$ standard deviations measured in a panel of 30 sera from healthy donors and 45 sera from patients with inflammatory bowel disease who were treatment naïve for VDZ.

\section{Endpoints and Definitions}

Clinical outcomes were clinical response (>70-point decrement in CDAI from baseline) and corticosteroid-free clinical remission (CDAI $<150$ points and no corticosteroid use). Biochemical remission was defined as a serum CRP concentration $<5 \mathrm{mg} / \mathrm{L}$ measured in patients with increased CRP at the start of the study. Endoscopic remission and response were defined as a SES-CD score $<4$ and a $\geq 50 \%$ reduction score compared with baseline, respectively. ${ }^{23,24}$ Histological remission was defined as GS $<3.1$ (which correlates with absence of neutrophils in the epithelium $)^{18,25,26}$ and an RHI $\leq 6$ (absence of granulocyte in mucosal biopsies). ${ }^{19}$

\section{Statistical Analysis}

Patient characteristics were evaluated using descriptive statistics. All patients who received at least 1 dose of VDZ were included in the analysis. For patients who withdrew from the study before week 26 or week 52, the exit endoscopy score was analyzed. Missing VDZ serum concentrations were not imputed. VDZ concentration data were compared between patients with and without endoscopic remission and response, using the Wilcoxon-Mann-Whitney test. Fisher's exact test was used to compare endoscopic outcomes at week 26 across VDZ quartiles at week 22. Optimal cutpoints of VDZ concentrations associated with endoscopic outcomes were determined using receiver operating characteristic (ROC) curve analysis. Correlations among clinical, endoscopic, and histological outcomes were calculated using Pearson correlation for binary outcome $(\phi$ coefficient). Subgroup analyses were performed to assess how sensitive endoscopic remission rates at weeks 26 and 52 were to variations in the study population. Endoscopic remission at weeks 26 and 52 were stratified by anti-TNF use (anti-TNF naïve vs anti-TNF exposed patients), corticosteroid use at inclusion $(<20 \mathrm{mg}$ vs $\geq 20 \mathrm{mg} / \mathrm{d})$ and baseline CRP concentrations $(<5 \mathrm{mg} / \mathrm{L}$ vs $\geq 5 \mathrm{mg} / \mathrm{L})$. All statistical testing was performed at the .05 significance level using $\mathrm{R}$ version 3.4.3 (R Foundation for statistical computing, Vienna, Austria).

\section{Results}

\section{Patient Disposition and Demographics}

The disposition of the patients is shown in Supplementary Figure 1. Data from 110 participants were analyzed. Seventy-six patients completed week 26 of the study, and 74 of 76 underwent the week 26 endoscopy. Sixty-three patients completed week 52 and 61 of 63 had an endoscopic assessment at that time point. Fourteen patients of 34 early withdrawals before week 26 underwent an exit endoscopy at a median time point of 22.5 weeks. One patient of 11 early withdrawals between weeks 26 and 52 underwent an early withdrawal endoscopy, which was performed at week 28. In total, 260 endoscopic procedures were analyzed. Of these 260 videos, 69 needed a second review because of discrepant reads between SR and CR. The mismatch rate in SES-CD scores between SR and CR was $27 \%(69 / 260)$.

Patient characteristics and demographics are summarized in Table 1 . The mean baseline CDAI score was 261, and the mean SES-CD score was 12 . Nine (8\%) of 110 patients had actively draining fistulas at baseline and all fistulas were perianal. In these 9 patients, the rectum was inflamed (according to the SES-CD score) in 2 patients, and both patients did not show endoscopic improvement in the rectum at week 26 or at week 52 . In these 2 patients, there were no changes observed regarding fistula drainage (assessed clinically) at both time points. For all 9 patients, endoscopic remission was observed in 33\% (3/9) and 22\% (2/9) at week 26 and 52, respectively. Fistula closure, as assessed clinically, was observed in 3 of 9 patients during 52 weeks of follow-up. Concomitant treatment with corticosteroids and immunosuppressives was present in $41 \%$ and $20 \%$ of patients at study entry, respectively, and $88 \%$ had previously been treated with TNF antagonists. Twenty patients (18\%) of 110 were using prednisone at baseline, 8 of whom received "high" prednisone doses defined as $\geq 20$ $\mathrm{mg} / \mathrm{d}$. Sixty-eight percent of patients (75/110) had elevated CRP levels ( $\geq 5 \mathrm{mg} / \mathrm{L})$ at baseline. Sixty-four percent $(70 /$ 110 ) of patients received an additional infusion with VDZ at week 10 because of lack of clinical response.

\section{Clinical Outcomes}

Corticosteroid-free clinical remission was observed in 32 (29\%) of 110 patients at week 26 and in 34 (31\%) of 110 at week 52 (Figure 1). Clinical response was present in 42 (38\%) of 110 patients at week 26 and in 39 (35\%) of 110 at week 52 (Figure 1). The mean drop in CDAI score from 
Table 1.Patient Characteristics at Baseline

\begin{tabular}{|c|c|}
\hline Total & 110 \\
\hline Age, $y$ & $36(28-46)$ \\
\hline Female, n (\%) & $77(70)$ \\
\hline BMI, $\mathrm{kg} / \mathrm{m}^{2}$ & $24(22-27)$ \\
\hline \multicolumn{2}{|l|}{ Smoking status } \\
\hline Current smoker & $30(27)$ \\
\hline Ex-smoker & $33(30)$ \\
\hline Never smoked & $47(43)$ \\
\hline Age at diagnosis, yr & $23(19-34)$ \\
\hline Disease duration, yr & $9(5-16)$ \\
\hline \multicolumn{2}{|l|}{ Disease location, n (\%) } \\
\hline lleitis & $26(24)$ \\
\hline Colitis & $32(30)$ \\
\hline Ileocolitis & $50(46)$ \\
\hline Draining fistulas, $\%$ & $9(8)$ \\
\hline Prior anti-TNF therapy, $\mathrm{n}(\%)$ & $97(88)$ \\
\hline High sensitive CRP, mg/L & $9(4-22)$ \\
\hline Elevated CRP at baseline, $\mathrm{n}(\%)$ & $75(71 \%)$ \\
\hline Serum albumin $(g / d L)$ & $42(38-44)$ \\
\hline CDAI & $261(238-312)$ \\
\hline SES-CD & $12(7-17)$ \\
\hline Concomitant corticosteroids, n (\%) & $45(41)$ \\
\hline Concomitant immunomodulators, $\mathrm{n}(\%)$ & $22(20)$ \\
\hline
\end{tabular}

NOTE. values are median (interquartile range) or $\mathrm{n}(\%)$. $\mathrm{BMI}$, body mass index.

baseline to week 26 and 52 was 131.52 and 124.83 points, respectively. CDAI outcomes (ie, proportion of patients with clinical remission and response) and normalization of CRP concentrations (CRP $<5 \mathrm{mg} / \mathrm{L})$ at all time points are presented in Supplementary Figure 2.

\section{Endoscopic and Histological Outcomes}

In the nonresponder imputation population (110 patients), endoscopic response was observed in $44(40 \%)$ of 110 patients at week 26 (95\% confidence interval [CI] $0.31-0.50)$ and in 50 (45\%) of 110 at week 52 (95\% CI 0.36-0.55) (Figure 1). Endoscopic remission was present in 36 (33\%; 95\% CI 0.24-0.42) and in 40 (36\%; 95\% CI: $0.28-0.46$ ) of 110 patients at week 26 and week 52, respectively. The mean change in SES-CD scores from baseline to week 26 and 52 were 4.05 and 4.65, respectively. Clinical and endoscopic outcomes in a subset of patients $(n=70)$ who received an additional week 10 infusion with VDZ are shown in Supplementary Figure 3. The effect of prior exposure to TNF antagonists on these outcomes could not be assessed, because most patients received prior treatment with these agents. Endoscopic remission at week 26 did not differ between terminal ileum and colonic segments (Supplementary Figure 4). The proportion of patients who had documented endoscopic lesions at baseline and achieved endoscopic remission at week 26 , was $66 \%, 62 \%$, $74 \%, 63 \%$, and $77 \%$ in the terminal ileum, ascending colon, transverse colon, descending colon and sigmoid, and rectum, respectively. Endoscopic remission rates at weeks 26 and 52 were analyzed across disease duration quartiles (ie, $<5$ years, between 5 and 9 years, between 9 and 15.5 years, and $>15.5$ years). Higher endoscopic remission rates at week 52 were observed in patients with a shorter disease duration compared with patients with a longer disease course (Supplementary Figure 5). This differences was not observed at week 26. Several sensitivity analyses were performed, as shown in Supplementary Figure 6. Eightyeight percent of patients $(97 / 110)$ were exposed to prior anti-TNF treatment and endoscopic remission was achieved in 29\% (CI 20-39) and 33\% (CI 24-43) at week 26 and 52, respectively. Sixty-eight percent of patients (75/110) had elevated CRP levels ( $\geq 5 \mathrm{mg} / \mathrm{L}$ ) at baseline, and in this subgroup endoscopic remission at weeks 26 and 52 was achieved in 25\% (CI 16-37) and 28\% (CI 19-40), respectively. Eighteen percent of patients $(20 / 110)$ used prednisone at baseline ( 8 at high $[\geq 20 \mathrm{mg} / \mathrm{d}]$ dose). Endoscopic remission was achieved in $25 \%$ of prednisone users at baseline at weeks 26 and 52 .

Analysis of histologic outcomes was restricted to paired biopsies from all segments collected at week 0 and week 26 with active inflammation at baseline. In total, 132 paired biopsies in 65 patients (ie, collected at week 0 and week 26 obtained from the same segment) were analyzed in which active inflammation was present at baseline. Sixty-seven biopsies had active inflammation at baseline according to the GS (score $\geq 3.1$ ), and 43 (64\%) of 67 showed histological remission (GS $<3.1$ ) at week 26 (Figure $2 A$ ). Fifty-six biopsies had active inflammation at baseline according to the RHI (score $>7$ ), and histological remission (RHI $\leq 6$ ) was observed in 37 (66\%) of 56 biopsies at week 26 (Figure $2 A$ ). The mean change in RHI scores from baseline to week 26 was 2.86. Next, correlations between endoscopic and histological remission at week 26 were analyzed. For 125 samples (64 patients), SES-CD and GS outcomes were available that were scored in the same segments. In $88 \%$ (78/89) of these samples, which were obtained from patients achieving endoscopic remission at week 26, histological remission was also observed $(\phi=0.45)$. For 130 samples (65 patients), SES-CD and RHI scores were available in the same segments. In 91\% (84/92) of these samples achieving endoscopic remission at week 26, histological remission was present $(\phi=0.46)$ (Supplementary Table 1). Q4 In addition, correlations between clinical remission and endoscopic and histological outcomes were analyzed. Clinical remission was observed in 36\% (13/36) of patients who also achieved endoscopic remission at week $26(\phi=$ $0.11)$. In $47 \%(18 / 38)$ and $50 \%(21 / 42)$ of patients who achieved clinical remission, histological remission at week 26 was observed using the GS $(\phi=0.10)$ and RHI $(\phi=$ 0.19), respectively (Supplementary Table 2).

\section{Pharmacokinetic/Pharmacodynamic Analysis}

VDZ serum trough concentrations up to week 22 are depicted in Supplementary Figure 7. The median VDZ serum concentration was significantly higher at all time points except for weeks 2 and 14 in patients who achieved endoscopic remission than in those who did not (Figure 3). In patients with and without endoscopic remission, median VDZ serum concentrations were 28 and $26 \mathrm{mg} / \mathrm{L}$ at week 2 
Figure 1. Proportion of paclinical remission (CDAl $<150$ ), clinical response (at least 70-point drop in CDAI compared with baseline), endoscopic remission (SES-CD <4) and endoscopic response $(50 \%$ reduction in SES-CD compared with baseline) at week 26 and week 52. Proportions were determined in nonresponder imputation population $(n=110)$. tients achieving steroid-free

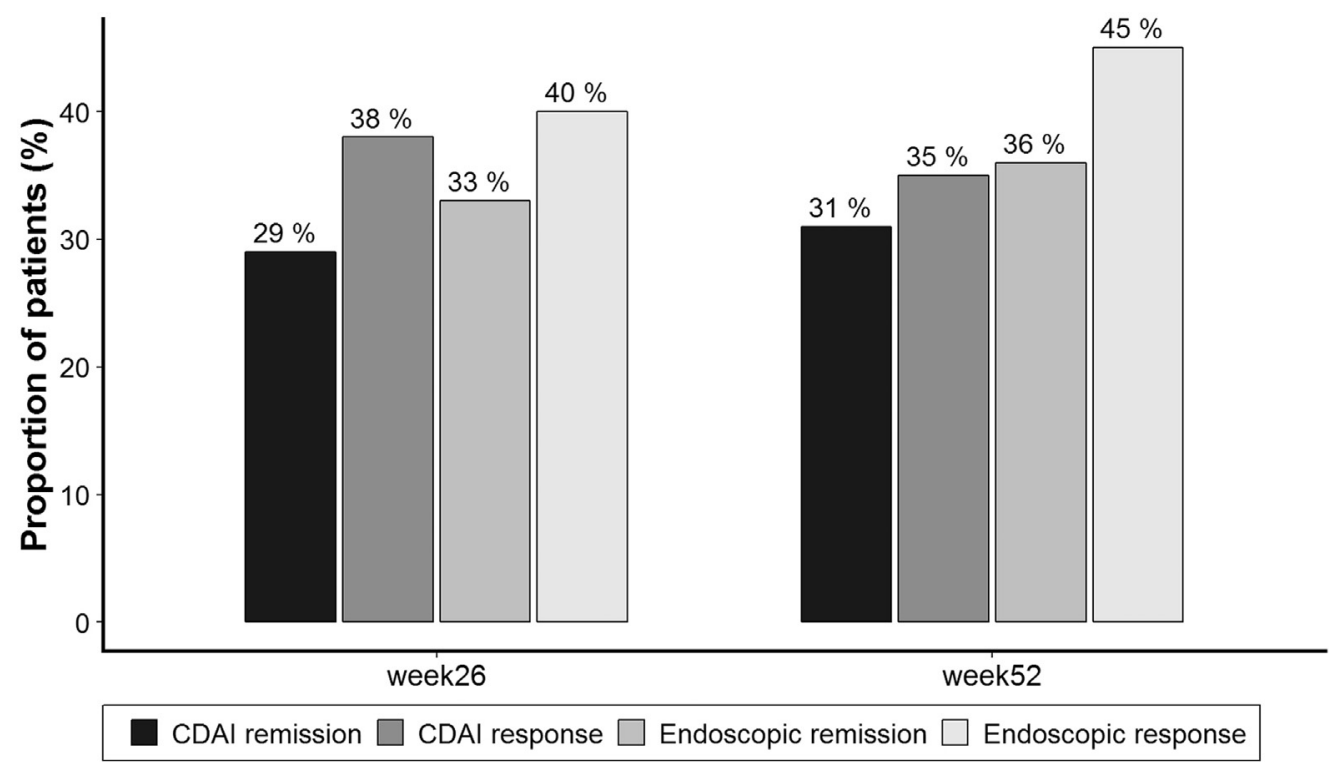

$(P=.12), 31$ and $25 \mathrm{mg} / \mathrm{L}$ at week $6(P=.01), 31$ and $26 \mathrm{mg} / \mathrm{L}$ at week $10(P=.04), 25$ and $22 \mathrm{mg} / \mathrm{L}$ at week 14 $(P=.29)$, and 18 and $11 \mathrm{mg} / \mathrm{L}$ at week $22(P=.0005)$. Similarly, patients with endoscopic response at week 26 had significantly higher median VDZ concentrations at these same time points (Figure $3 C$ ). Median VDZ serum concentrations were 27 and $26 \mathrm{mg} / \mathrm{L}$ at week $2(P=.43), 31$ and $24 \mathrm{mg} / \mathrm{L}$ at week $6(P=.02), 31$ and $25 \mathrm{mg} / \mathrm{L}$ at week 10 $(P=.02), 26$ and $21 \mathrm{mg} / \mathrm{L}$ at week $14(P=.10)$, and 17 and $11 \mathrm{mg} / \mathrm{L}$ at week $22(P=.01)$ in patients with and without endoscopic response, respectively.

In addition, the concentration-effect relationship between VDZ serum concentrations at week 22 (ie, serum concentration at trough closest to the week 26 colonoscopy) and endoscopic outcomes at week 26, were analyzed by quartile analysis (Figure 4). Higher VDZ serum concentrations at week 22 were associated with higher rates of endoscopic remission (Figure $4 A$ ) and endoscopic response (Figure $4 B$ ). Endoscopic remission rates at week 26 were
$16 \%, 56 \%, 47 \%$, and $75 \%$ among patients in quartile 1 through quartile 4 , respectively $(P=.004)$. Endoscopic response rates at week 26 were $32 \%, 56 \%, 58 \%$, and $75 \%$ among patients in quartile 1 through quartile 4 , respectively $(P=.08)$.

The area under the ROC curve, quantifying associations between VDZ serum levels at week 22 and endoscopic remission and response at week 26, is shown in Figure 5. A VDZ serum concentration above the cutoff level of $10 \mathrm{mg} / \mathrm{L}$ at week 22 correlated with endoscopic remission. A VDZ cutoff serum concentration of $10 \mathrm{mg} / \mathrm{L}$ at week 22 was identified that best discriminated patients with and without endoscopic remission at week 26, with an area under the curve of 0.74 , a sensitivity and specificity of 0.91 and 0.54 , respectively, a positive predictive value of $64 \%$, and a negative predictive value of $87 \%$ (Figure $4 C$ ). A VDZ Q6 threshold concentration of $10.5 \mathrm{mg} / \mathrm{L}$ at week 22 discriminated patients with and without endoscopic response at week 26 with an area under the curve of 0.69 with a
Figure 2. $(A)$ Proportion of biopsies with histological remission at week 26, defined as GS $<3.1$ and $\mathrm{RHI} \leq 6$. Paired biopsies (week 0 and week 26) were analyzed with documented active inflammation at baseline. (B) Histologic images before treatment with VDZ, showing colonic mucosa with active inflammation and ulceration (top), and 26 weeks following VDZ treatment (bottom) showing normal colonic mucosa.

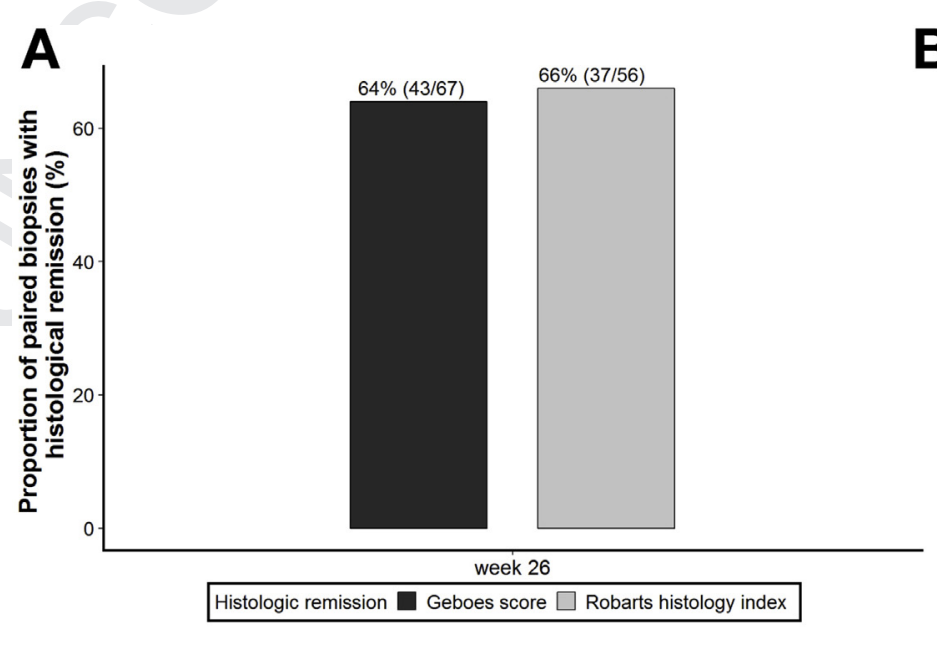

B

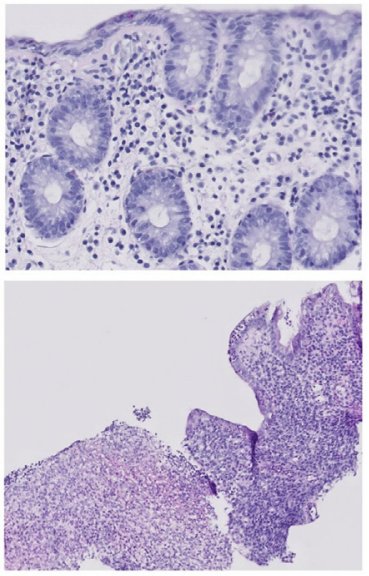

541

542

543

544

545

546

547

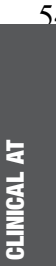

558

559

560

561

562

563

564

565

566

567

568

569

570

571

572

573

574

575

576

577

578

579

580

581

582

583

584

585

586

587

588

589

590

591

592

593

594

595

596

597

598

599

600 
601

602

603

604

605

606

607

608

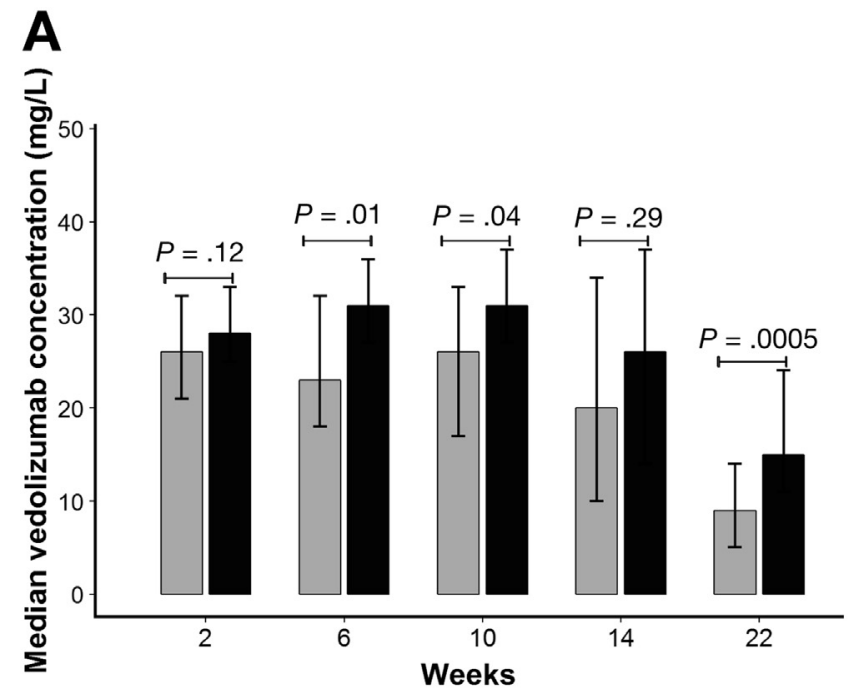

Endoscopic remission $\square$ No (n=39) $\square$ Yes ( $n=35)$

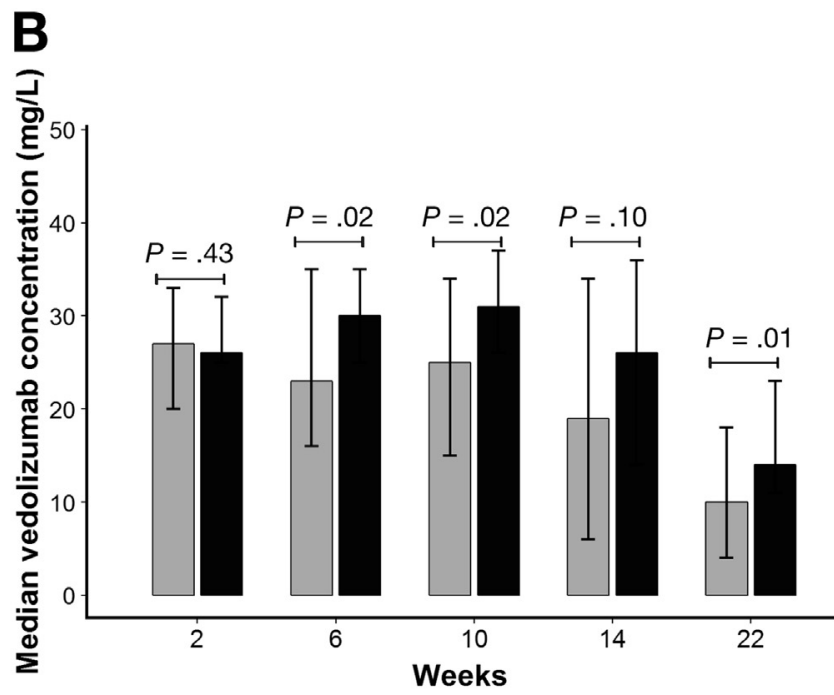

Endoscopic response $\square$ No (n=34) $\square$ Yes ( $n=40)$

Figure 3. (A) Median VDZ serum concentrations at different time points among patients who did (blue) or did not (red) achieve endoscopic remission (SES-CD $<4$ ) at week 26. (B) Median VDZ serum concentrations at different time points among patients with (blue) and without (red) endoscopic response (decrease in SES-CD $>50 \%$ ) at week 26 . Seventy-four procedures were performed at week 26. $P$ values were calculated based on Wilcoxon-Mann-Whitney test.

positive predictive value and negative predictive value of $69 \%$ and $70 \%$, respectively, with a sensitivity of 0.79 and a specificity of 0.59 (Figure $4 D$ ). Supplementary Tables 3 and 4 show correlations between VDZ serum concentrations at week 2 , week 6 , and week 14 and endoscopic remission and response at week 26 .

\section{Immunogenicity}

The proportion of patients with detectable AVA at different time points throughout the study varied between
$1 \%$ and $4 \%$ (Supplementary Table 5). All patients with AVA had measurable VDZ serum concentrations; median VDZ serum concentrations in patients with detectable AVA were $26,26,26,21.5$, and $12 \mathrm{mg} / \mathrm{L}$ at week 2 , week 6 , week 10 , and week 22, respectively. AVA disappeared in all patients except for 1 patient who had detectable AVA up to week 52 , but VDZ treatment was not discontinued in this patient. One patient with detectable AVA discontinued VDZ treatment before week 26 , because of insufficient clinical and endoscopic response.
661

662

663

664

665

666

667

668

669

670

671

672

673

674

675

676

677

678

679

680

681

682

683

684

685

686

687

688

689

690

691

692

693

694

695

696

697

698

699

700

701

702

703

704

705

706

707

708

709

710

711

712

713

714

715

716

717

718

719

720 


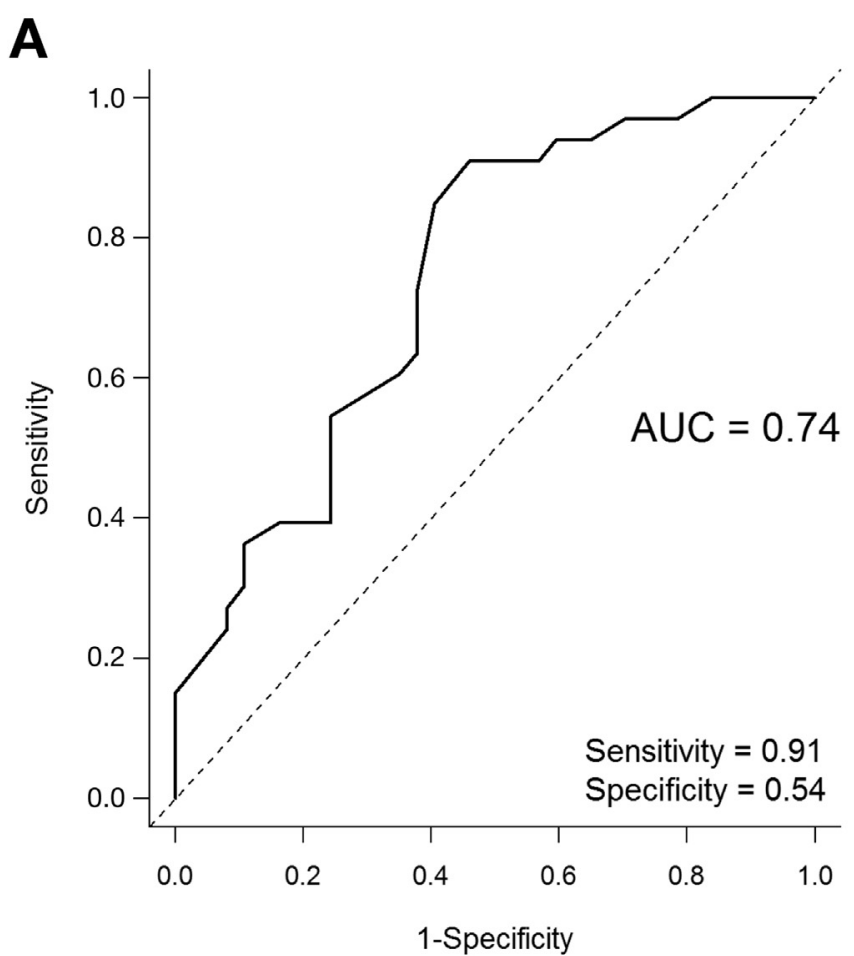

Figure 5. (A) ROC curve analysis: correlations between VDZ serum levels at week 22 and endoscopic remission at week 26. (B) ROC curve analysis: correlations between VDZ serum levels at week 22 and endoscopic response at week 26. AUC, area under curve.

\section{Safety}

Adverse events that occurred in at least $5 \%$ of patients receiving VDZ are listed in Supplementary Table 6. Skinrelated problems, headache, and nasopharyngitis were among the most frequently reported side effects. Two serious adverse events were reported. One patient underwent a subtotal colectomy with ileosigmoidal anastomosis because of an intestinal stenosis and 1 patient was admitted for worsening of symptoms consistent with a flare of CD. Three of 110 patients discontinued treatment because of side effects. No cases of Clostridium difficile were observed. Twenty-five of 110 patients reported arthralgia/arthritis complaints ( 21 were classified as mild, 4 as moderate) by week 52 , and 4 of 25 were reported as related to VDZ. There were 5 reports of worsening of arthralgia, 2 of 5 were reported as related to the treatment.

\section{Discussion}

The patients evaluated in LOVE-CD were a difficult-totreat population with an average disease duration of 9 years. Furthermore, most participants (88\%) had previously failed treatment with TNF antagonists. Corticosteroid-free clinical remission was observed in $29 \%$ and $31 \%$ of these patients following 26 and 52 weeks of VDZ therapy, respectively, and clinical response was present in 38\% and $35 \%$ at these time points. By way of comparison, in GEMINI $3,27 \%$ of TNF-exposed patients achieved clinical remission by week 10 . Thus, our findings are consistent with previously published data on the beneficial effect of VDZ induction therapy on clinical disease activity in $\mathrm{CD}$.

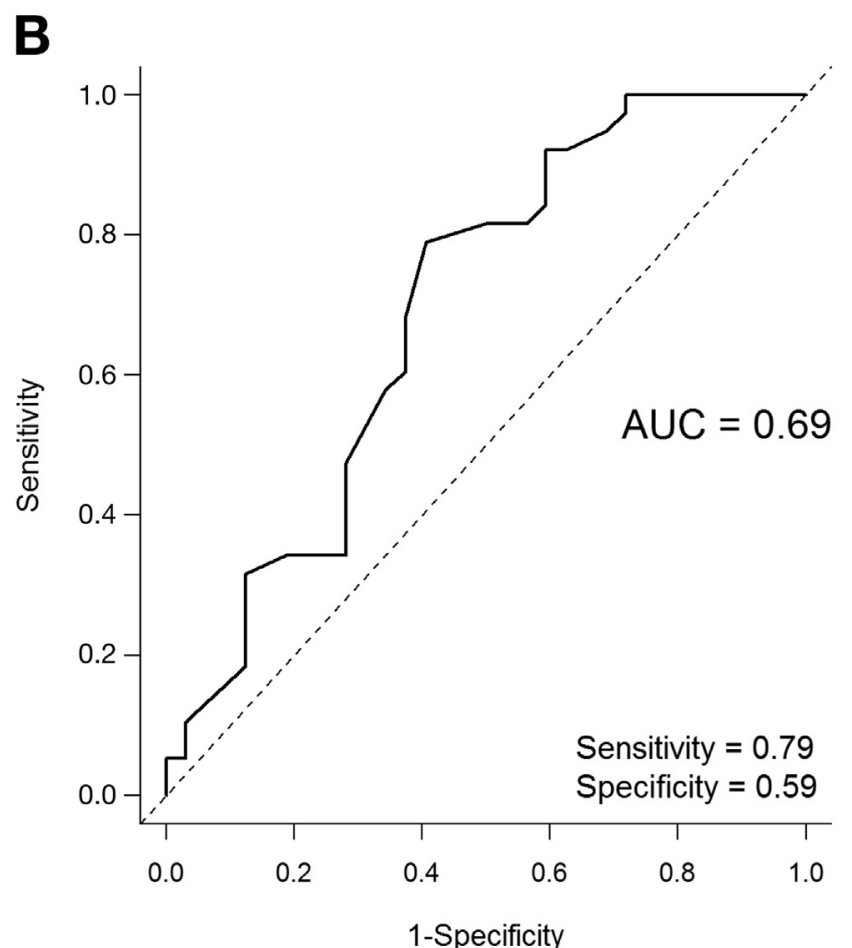

781

782

783

784

785

786

787

788

798

799

800

801

802

803

804

805

806

807

808

809

810

811

812

813

814

815

816

817

818

819

820

821

822

823

824

825

826

827

828

829

830

831

832

833

834

835

836

837

838

839

840 
protocol and patient population factors may account for these differences. Most importantly, in LOVE-CD dose intensification, consisting of administration of a week 10 VDZ infusion, was specified by the protocol if clinically relevant improvement in disease activity was not observed at that time. This finding should lead to further controlled studies of intensification of VDZ induction therapy in treatment-resistant patients.

It is relevant to consider that the results of these 2 openlabel studies can be benchmarked against estimates derived from a recent meta-analysis of placebo-controlled trials that used SES-CD to define endoscopic remission and response rates. In this study, the pooled endoscopic remission and response rates using the same definitions used in LOVE-CD and VERSIFY were 5.2 (95\% CI 1.7\%-8.8\%) and $16.2 \%$ (95\% CI 10.5\%-22\%), respectively (Duijvestein $\mathrm{M}$ et al, manuscript submitted to Clinical Gastroenterology and Hepatology). Thus, the endoscopic rates of remission and response were approximately twice those expected with placebo. In contrast, a recently reported open-label trial of mongersen, an antisense oligonucleotide against Smad7 performed in patients with moderate to severe CD, reported remission and response rates, using the same SES-CD definitions, of only $4 \%$ and $15 \%$ of patients, respectively. ${ }^{29}$ Subsequently, the mongersen development program was terminated following an interim analysis of the results of 2 independent placebo-controlled trials that showed no benefit of the drug on either clinical or endoscopic outcome. Collectively, these effects on mucosal inflammation observed in LOVE-CD were the results of VDZ therapy and not due to regression to the mean or the presence of concomitant therapy.

Although indirect comparisons between these results and those obtained for other drug classes should be interpreted with considerable caution, they are unavoidable in the absence of comparative effectiveness trials. The endoscopic remission rate of $33 \%$ at week 26 and $36 \%$ at week 52 that we report in the current study compares favorably with those reported in clinical trials with other treatments for CD. In the EXTEND trial with adalimumab, $27 \%$ and $24 \%$ of the patients had no ulcerations at week 12 and 52, respectively. $^{27}$ In the SONIC trial, patients also had an endoscopic evaluation at week 26 and a less refractory population of patients with CD was studied, because they were all naïve to immunosuppressive agents and biologics at study entry. ${ }^{30}$ In this study, "absence of ulcers" was observed in $30 \%$ on infliximab monotherapy and in $44 \%$ on combination treatment. More recently the IMMUNITI investigators reported endoscopic remission and response rates of $13 \%$ and $17 \%$ following 44 weeks of ustekinumab therapy in a group of patients with a moderately high rate of previous failure to TNF antagonists who had responded to ustekinumab induction therapy. ${ }^{31}$ Finally, in a recent trial of risankizumab, a novel antibody directed against the p19 subunit of interleukin 23, relatively higher doses of antibody (600 mg every 4 weeks) were administered during 2 consecutive induction phases followed by $180 \mathrm{mg}$ every 8 weeks subcutaneously. ${ }^{32}$ After 1 year of treatment, endoscopic remission was seen in $52 \%$ of the patients, which appears considerably higher than with ustekinumab. Whether it is the selective interleukin 23 inhibition or the more optimal exposure regimen that explains this difference remains to be clarified.

Collectively, this experience shows that endoscopic remission and response rates are relatively low even with our best investigational and established treatments, and that early treatment in biologically naïve patients provides the greatest opportunity for success. Further research into strategies for improving mucosal healing rates is a research priority. In this regard, our data evaluating drug concentrations is of interest. Overall, we found higher endoscopic remission and response rates in patients with higher serum concentrations, except for endoscopic outcomes at week 14, in which no significant differences in serum levels were found between patients with and without endoscopic remission and response at week 26 . This can be explained by the fact that week $14 \mathrm{VDZ}$ serum concentrations came from patients with and without an additional week 10 infusion (64\% vs 36\%, respectively). Endoscopic outcomes at week 26 were notably greater in the 3 highest quartiles of VDZ exposure than in the first quartile. Quartile analysis of exposure at week 22 indicated that a drug concentration above $7.6 \mu \mathrm{g} / \mathrm{mL}$ was associated with endoscopic healing; however, it remains to be determined whether this relationship is causal and if dose intensification will lead to better endoscopic outcomes. Hence, future prospective dose-optimization studies will have to prove if dose intensification can further improve outcomes. In addition, ROC curve analysis identified a VDZ serum cutoff level of $10 \mathrm{mg} /$ $\mathrm{L}$ at week 22 that correlated with optimal endoscopic outcomes at week 26 , with a high sensitivity (0.91), but with a rather low specificity (0.54). Correlations between VDZ serum concentrations at earlier time points (weeks 2, 6, and 14) and endoscopic outcomes at week 26 were less evident, suggesting that TDM may be useful only during mainte- Q9 nance therapy with VDZ.

Based on sensitivity analyses, no firm conclusions can be drawn as to which patient subgroups the observed outcomes apply. This is because most patients failed on prior anti-TNF treatment, prednisone was used in only $18 \%$ of patients at inclusion, and baseline CRP levels were elevated in most patients. We could not stratify patient outcomes based on estimates of disease burden, such as stool markers of inflammation or disease extent at imaging, because these analyses were not part of the study protocol.

Although endoscopic remission is associated with better outcomes in CD, less is known about the clinical relevance of achieving histological remission, which is emerging as a novel endpoint in clinical trials. ${ }^{33}$ Furthermore, endoscopic remission is not always paralleled by histological remission. $^{34}$ Although validated definitions for histological remission in $\mathrm{CD}$ are lacking, ${ }^{35}$ we believe that we used the best available scores determined by a central independent reader. Histological remission, an outcome not previously reported in VDZ studies in CD, was observed in 64\% and $66 \%$ of paired biopsies at week 26 . Interestingly, in approximately $90 \%$ of samples, a correlation was found between endoscopic and histological remission at week 26 .
901

902

903

904

905

906

907

908

909

910

911

912

913

914

915

916

917

918

919

920

921

922

923

924

925

926

927

928

929

930

931

932

933

934

935

936

937

938

939

940

941

942

943

944

945

946

947

948

949

950

951

952

953

954

955

956

957

958

959

960 
Hence, these findings highlight the fact that VDZ is able to induce histological remission in endoscopic responders in CD. Whether histological remission will result in improved long-term outcomes needs to be defined.

Between $1 \%$ and $4 \%$ of patients had detectable AVA at different time points throughout the study. Of note, AVAs seem to be transient in most cases, unrelated to clinical outcomes and they did not impact VDZ serum concentrations. From these observations, it can be concluded that determination of AVA is not clinically useful with the assay that we used, which is in line with observations by others. $^{36-38}$

In conclusion, this study shows that VDZ is effective in achieving endoscopic and histological remission in CD, and improved endoscopic outcomes are associated with higher VDZ serum concentrations.

\section{Supplementary Material}

Note: To access the supplementary material accompanying this article, visit the online version of Gastroenterology at www.gastrojournal.org, and at https://doi.org/10.1053/ j.gastro.2019.05.067.

\section{References}

1. Baumgart DC, Sandborn WJ. Crohn's disease. Lancet 2012;380:1590-1605.

2. Sandborn WJ, Feagan BG, Rutgeerts $P$, et al. Vedolizumab as induction and maintenance therapy for Crohn's disease. N Engl J Med 2013;369:711-721.

3. Sands BE, Feagan BG, Rutgeerts $P$, et al. Effects of vedolizumab induction therapy for patients with Crohn's disease in whom tumor necrosis factor antagonist treatment failed. Gastroenterology 2014;147:618-627.e3.

4. Noman M, Ferrante M, Bisschops R, et al. Vedolizumab induces long-term mucosal healing in patients with Crohn's disease and ulcerative colitis. J Crohns Colitis 2017;11:1085-1089.

5. Dulai PS, Singh S, Jiang X, et al. The real-world effectiveness and safety of vedolizumab for moderate-severe Crohn's disease: results from the US VICTORY Consortium. Am J Gastroenterol 2016;111:1147-1155.

6. Schreiber S, Dignass A, Peyrin-Biroulet L, et al. Systematic review with meta-analysis: real-world effectiveness and safety of vedolizumab in patients with inflammatory bowel disease. J Gastroenterol 2018;53:1048-1064.

7. Engel T, Ungar B, Yung DE, et al. Vedolizumab in IBD-lessons from real-world experience; a systematic review and pooled analysis. J Crohns Colitis 2018; 12:245-257.

8. De Vos M, Dhooghe B, Vermeire S, et al. Efficacy of vedolizumab for induction of clinical response and remission in patients with moderate to severe inflammatory bowel disease who failed at least two TNF antagonists. United European Gastroenterol J 2018;6:439-445.

9. Ferrante M, Colombel JF, Sandborn WJ, et al. Validation of endoscopic activity scores in patients with Crohn's disease based on a post hoc analysis of data from SONIC. Gastroenterology 2013;145:978-986.e5.
10. Colombel JF, Narula N, Peyrin-Biroulet L. Management strategies to improve outcomes of patients with inflammatory bowel diseases. Gastroenterology 2017;152: 351-361.e5.

11. Peyrin-Biroulet L, Sandborn W, Sands BE, et al. Selecting Therapeutic Targets in Inflammatory Bowel Disease (STRIDE): determining therapeutic goals for treat-totarget. Am J Gastroenterol 2015;110:1324-1338.

12. Adedokun OJ, Sandborn WJ, Feagan BG, et al. Association between serum concentration of infliximab and efficacy in adult patients with ulcerative colitis. Gastroenterology 2014;147:1296-1307.e5.

13. Papamichael K, Van Stappen $T$, Vande Casteele $N$, et al. Infliximab concentration thresholds during induction therapy are associated with short-term mucosal healing in patients with ulcerative colitis. Clin Gastroenterol Hepatol 2016;14:543-549.

14. Gibson DJ, Heetun ZS, Redmond CE, et al. An accelerated infliximab induction regimen reduces the need for early colectomy in patients with acute severe ulcerative colitis. Clin Gastroenterol Hepatol 2015;13:330-335.e1.

15. Rosario M, French JL, Dirks NL, et al. Exposure-efficacy relationships for vedolizumab induction therapy in patients with ulcerative colitis or Crohn's disease. J Crohns Colitis 2017;11:921-929.

16. Daperno M, D'Haens G, Van Assche G, et al. Development and validation of a new, simplified endoscopic activity score for Crohn's disease: the SES-CD. Gastrointest Endosc 2004;60:505-512.

17. Reinisch W, Mishkin DS, Oh YS. P132 analysis of various central endoscopy reading methodologies in the BERGAMOT exploratory induction cohort evaluating etrolizumab in Crohn's disease. J Crohns Colitis 2018:S161.

18. Geboes K, Riddell R, Ost A, et al. A reproducible grading scale for histological assessment of inflammation in ulcerative colitis. Gut 2000;47:404-409.

19. Mosli MH, Feagan BG, Zou G, et al. Development and validation of a histological index for UC. Gut 2017; 66:50-58.

20. Rispens T, Leeuwen A, Vennegoor A, et al. Measurement of serum levels of natalizumab, an immunoglobulin G4 therapeutic monoclonal antibody. Anal Biochem 2011; 411:271-276.

21. Rispens $\mathrm{T}$, de Vrieze $\mathrm{H}$, de Groot $\mathrm{E}$, et al. Antibodies to constant domains of therapeutic monoclonal antibodies: anti-hinge antibodies in immunogenicity testing. $\mathrm{J}$ Immunol Methods 2012;375:93-99.

22. Wolbink GJ, Vis M, Lems W, et al. Development of antiinfliximab antibodies and relationship to clinical response in patients with rheumatoid arthritis. Arthritis Rheum 2006;54:711-715.

23. Vuitton L, Marteau P, Sandborn WJ, et al. IOIBD technical review on endoscopic indices for Crohn's disease clinical trials. Gut 2016;65:1447-1455.

24. Vermeire S, Schreiber S, Petryka R, et al. Clinical remission in patients with moderate-to-severe Crohn's disease treated with filgotinib (the FITZROY study): results from a phase 2, double-blind, randomised, placebo-controlled trial. Lancet 2017;389:266-275.
1021

1022

1023

1024

1025

1026

1027

1028 
25. Bessissow T, Lemmens B, Ferrante M, et al. Prognostic value of serologic and histologic markers on clinical relapse in ulcerative colitis patients with mucosal healing. Am J Gastroenterol 2012;107:1684-1692.

26. Kleer CG, Appelman HD. Ulcerative colitis: patterns of involvement in colorectal biopsies and changes with time. Am J Surg Pathol 1998;22:983-989.

27. Rutgeerts $P$, Van Assche G, Sandborn WJ, et al. Adalimumab induces and maintains mucosal healing in patients with Crohn's disease: data from the EXTEND trial. Gastroenterology 2012;142:1102-1111.e2.

28. Danese S, Feagan B, Sandborn W. OP023 A phase 3b open-label multicentre study (VERSIFY) of the efficacy of vedolizumab on endoscopic healing in moderately to severely active Crohn's disease (CD). J Crohns Colitis 2018;12:S016-S017.

29. Feagan BG, Sands BE, Rossiter G, et al. Effects of mongersen (GED-0301) on endoscopic and clinical outcomes in patients with active Crohn's disease. Gastroenterology 2018;154:61-64.e6.

30. Colombel JF, Sandborn WJ, Reinisch W, et al. Infliximab, azathioprine, or combination therapy for Crohn's disease. N Engl J Med 2010;362:1383-1395.

31. Rutgeerts P, Gasink C, Chan D, et al. Efficacy of ustekinumab for inducing endoscopic healing in patients with Crohn's disease. Gastroenterology 2018;155:1045-1058.

32. Feagan BG, Panes J, Ferrante M, et al. Risankizumab in patients with moderate to severe Crohn's disease: an open-label extension study. Lancet Gastroenterol Hepatol 2018;3:671-680.

33. Romkens TE, Gijsbers K, Kievit W, et al. Treatment targets in inflammatory bowel disease: current status in daily practice. J Gastrointestin Liver Dis 2016;25:465-471.

34. Villanacci V, Antonelli E, Geboes K, et al. Histological healing in inflammatory bowel disease: a still unfulfilled promise. World J Gastroenterol 2013;19:968-978.

35. Novak G, Parker CE, Pai RK, et al. Histologic scoring indices for evaluation of disease activity in Crohn's disease. Cochrane Database Syst Rev 2017;7:CD012351.

36. Bian S, Dreesen E, Tang HT, et al. Antibodies toward vedolizumab appear from the first infusion onward and disappear over time. Inflamm Bowel Dis 2017; 23:2202-2208.

37. Ungar B, Kopylov U, Yavzori M, et al. Association of vedolizumab level, anti-drug antibodies, and alpha4beta7 occupancy with response in patients with inflammatory bowel diseases. Clin Gastroenterol Hepatol 2018; 16:697-705.e7.

38. Van den Berghe N, Verstockt B, Tops S, et al. Immunogenicity is not the driving force of treatment failure in vedolizumab-treated inflammatory bowel disease patients. J Gastroenterol Hepatol 2019;34:1175-1181.

\section{Received December 13, 2018. Accepted May 30, 2019.}

\section{Reprint requests}

Address requests for reprints to Geert D'Haens, MD, PhD, Amsterdam UMC University of Amsterdam, Department of Gastroenterology and Hepatology, Meibergdreef 9, 1100 DZ Amsterdam, The Netherlands. e-mail: g.dhaens@amc.uva.nl.

\section{Acknowledgments}

This study was an investigator-initiated study in Belgium and the Netherlands and was funded by Takeda Pharmaceuticals. We wish to acknowledge all participating investigators and study coordinators (sc). AMC: Prof $\mathrm{Dr} \mathrm{C}$ Ponsioen; Drs R. van den Berg; A. van der Linden (sc); N. Berkers (sc); W. Elmassaoudi (sc); J. de Jong (sc). Onze Lieve Vrouwe Gasthuis locatie oost: Drs J. Jansen; T. Schakel (sc). Erasmus MC: Dr B. Schultze; M. Polat-Utku (sc); H. van Santen (sc); S. de Bil (sc). Ziekenhuis Gelderse Vallei: Dr W. Mares; L. Homans (sc); Radboud MC: C. Slaa (sc); C. Jansen (sc). UZ Leuven: G. Schops (sc); L. Lapage (sc). Imelda ziekenhuis: Dr P. Bossuyt; K. Asnong (sc); I. van der Schoot (sc). UZ Gent: Dr M. de Vos; N. Swietek (sc); L. Sioncke (sc); AZ Sint Lucas Brugge: Dr P. van Hootegem; Hilde Nevens (sc); P. Vlieghe (sc); St Vincentius: Dr D. Staessen; A. Janssens (sc). AZ Damiaan: Dr G. Lambrecht; N. Rooryck (sc); B. Claerbout (sc). UZ Antwerpen: Dr E. Macken; E. Meersman (sc). ULB Erasme: C. Minsart (sc); V. Wambacq (sc); CHC Clinique Saint Joseph: Dr A. Colard; A. Deflndere (sc) ZNA Jan Palfijn: Dr J. Dutre; K. Bevers (sc). Ziekenhuis Oost Limburg: Dr P. Caenepeel; A. Eevers (sc). AZ Sint Lucas Gent: Prof H. Peeters; J. Goossens (sc); S. Claeys (sc). AZ Delta Roeselare: L. Decaesteker (sc); E. de Cock (sc). AZ Groeninge: Dr W. van Moerkercke; S. Himpe (sc). CHU Liege: Dr E. Louis; L. Boutaffala (sc); C. Guebelle (sc).

Author contributions: Study concept and design: ML, SV, FB, GD. Acquisition of clinical data: ML, SV, FH, DF, PB, PH, CJW, FB, GD. Analysis and interpretation of data: ML, SV, FH, PB, PH, SB, CA, RM, FB, GD. Drafting of the manuscript: ML, SV, GD; Critical revision of the manuscript for important intellectual content: FH, PB, SB, CA, RM; CJW, FB. Statistical analysis: NM. Administrative support: EC. Technical support: TR, AV.

\section{Conflicts of interest}

These authors disclose the following: Mark Löwenberg has served as speaker Q2 and/or principal investigator for AbbVie, Celgene, Covidien, Dr. Falk, Ferring Pharmaceuticals, Gilead, GlaxoSmithKline, Janssen-Cilag, Merck Sharp \& Dohme, Pfizer, Protagonist therapeutics, Receptos, Takeda, Tillotts, and Tramedico. He has received research grants from AbbVie, Merck Sharp \& Dohme, Achmea healthcare, and ZonMW. Severine Vermeire has received grant support from AbbVie, MSD, Pfizer, J\&J, and Takeda; received speaker fees from AbbVie, MSD, Takeda, Ferring, Dr. Falk Pharma Hospira, Pfizer Inc, and Tillots; and served as a consultant for AbbVie, MSD, Takeda, Ferring, Genentech/Roche, Robarts clinical trials, Gilead, Celgene, Prometheus, Avaxia, Prodigest, Shire, Pfizer Inc, Galapagos, Mundipharma, Hospira, Celgene, Second Genome, and Janssen. Frank Hoentjen has served on advisory boards, or as speaker or consultant for AbbVie, Celgene, Janssen-Cilag, MSD, Takeda, Celltrion, Teva, Sandoz, and Dr Falk, and has received grants from Dr Falk, Janssen-Cilag, and AbbVie. Denis Franchimont has received consultancy and lecture fees from MSD, AbbVie, Janssen, Pfizer, Takeda, Ferring, Falk, Mundipharma, Biogen, Amgen, and Merck Serono, and educational grants from MSD, AbbVie, Janssen, Takeda, Ferring, Mundipharma, Cook, and AstraZeneca. Peter Bossuyt has served as speaker for Takeda, AbbVie, Janssen, Pfizer, and Dr. Falk Pharma; has served as an advisor for MSD, Genetech Roche, Takeda, AbbVie, Mundipharma, Janssen, Dr. Falk Pharma, Pfizer, and Pentax; and has received grants from Janssens and AbbVie. Pieter Hindryckx has received consulting fees from AbbVie, Takeda, and Janssen; and speaker's fees from Ferring, Falk Pharma, Vifor Pharma, Tillotts Pharma, Chiesi, Takeda, and AbbVie. Theo Rispens has received honoraria for lectures from Pfizer, AbbVie, and Regeneron, and a research grant from Genmab. C. Janneke van der Woude received gran support from Falk Benelux and Pfizer; received speaker fees from AbbVie, Takeda, Ferring, Dr. Falk Pharma, Hospira, and Pfizer; and served as a consultant for AbbVie, MSD, Takeda, Celgene, Mundipharma, and Janssen. Ron Mathot has served as an advisor for Bayer, CSL Behring, Merck Sharp \& Dohme, Shire, and Zeria; and has received research grants from Bayer, CSL Behring, Shire, and ZonMW. Filip Baert has received consultancy and speakers fees from AbbVie, Celgene, MSD, Ferring, Janssen, Takeda, Pfizer/Hospira, and Mundipharma; and grants from AbbVie, Chiesi Farmaceutici, Ipsen, and Roche. Geert D'Haens has served as an advisor for AbbVie, Ablynx, Allergan, Amakem, Amgen, AM Pharma, Arena Pharmaceuticals, AstraZeneca, Avaxia, Biogen, BristolMyers Squibb, Boehringer Ingelheim, Celgene/Receptos, Celltrion, Cosmo, Covidien/Medtronics, Ferring, Dr Falk Pharma, Eli Lilly, Engene, Galapagos, Genentech/Roche, Gilead, Glaxo Smith Kline, Hospira/Pfizer, Immunic, Johnson \& Johnson, Lycera, Medimetrics, Millenium/Takeda, Mitsubishi Pharma, Merck Sharp Dome, Mundipharma, Nextbiotics, Novonordisk, Otsuka, Pfizer/Hospira, Photopill, Prometheus laboratories/ Nestle, Progenity, Protagonist, Robarts Clinical Trials, Salix, Samsung Bioepis, Sandoz, Seres/Nestle, Setpoint, Shire, Teva, Tigenix, Tillotts, Topivert, Versant, and Vifor; and received speaker fees from AbbVie, Biogen, Ferring, Johnson and Johnson, Merck Sharp Dome, Mundipharma, Norgine, Pfizer, Samsung Bioepis, Shire, Millenium/ Takeda, Tillotts, and Vifor. The remaining authors disclose no conflicts.

Funding

Unrestricted grant support from Takeda Pharmaceuticals.
1141

1142

1143

1144

1145

1146

1147

1148

1149

1150

1151

1152

1153

1154

1155

1156

1157

1158

1159

1160

1161

1162

1163

1164

1165

1166

1167

1168

1169

1170

1171

1172

1173

1174

1175

1176

1177

1178

1179

1180

1181

1182

1183

1184

1185

1186

1187

1188

1189

1190

1191

1192

1193

1194

1195

1196

1197

1198

1199

1200 


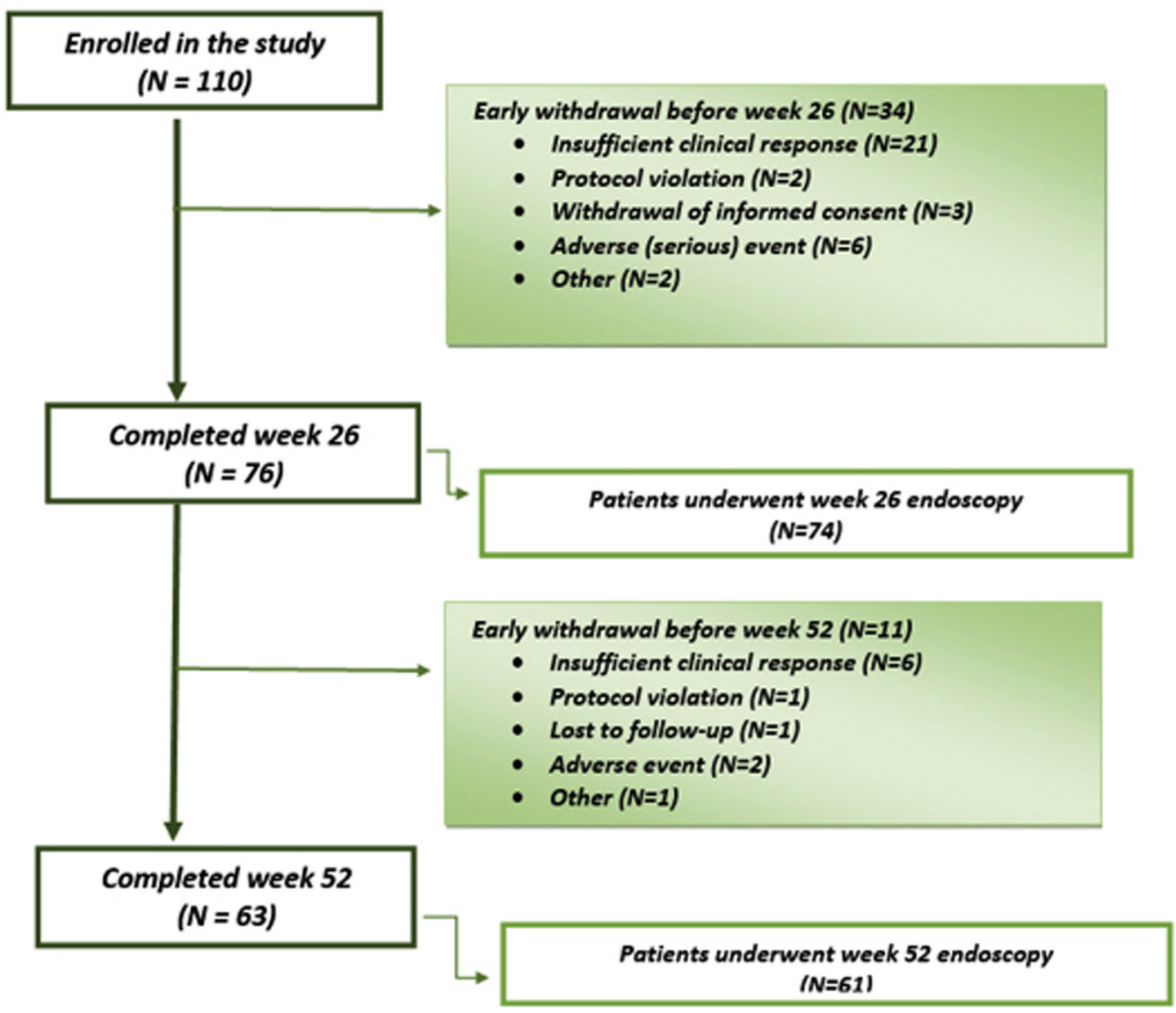

Supplementary Figure 1. Consort diagram: patient flowchart during the study. 


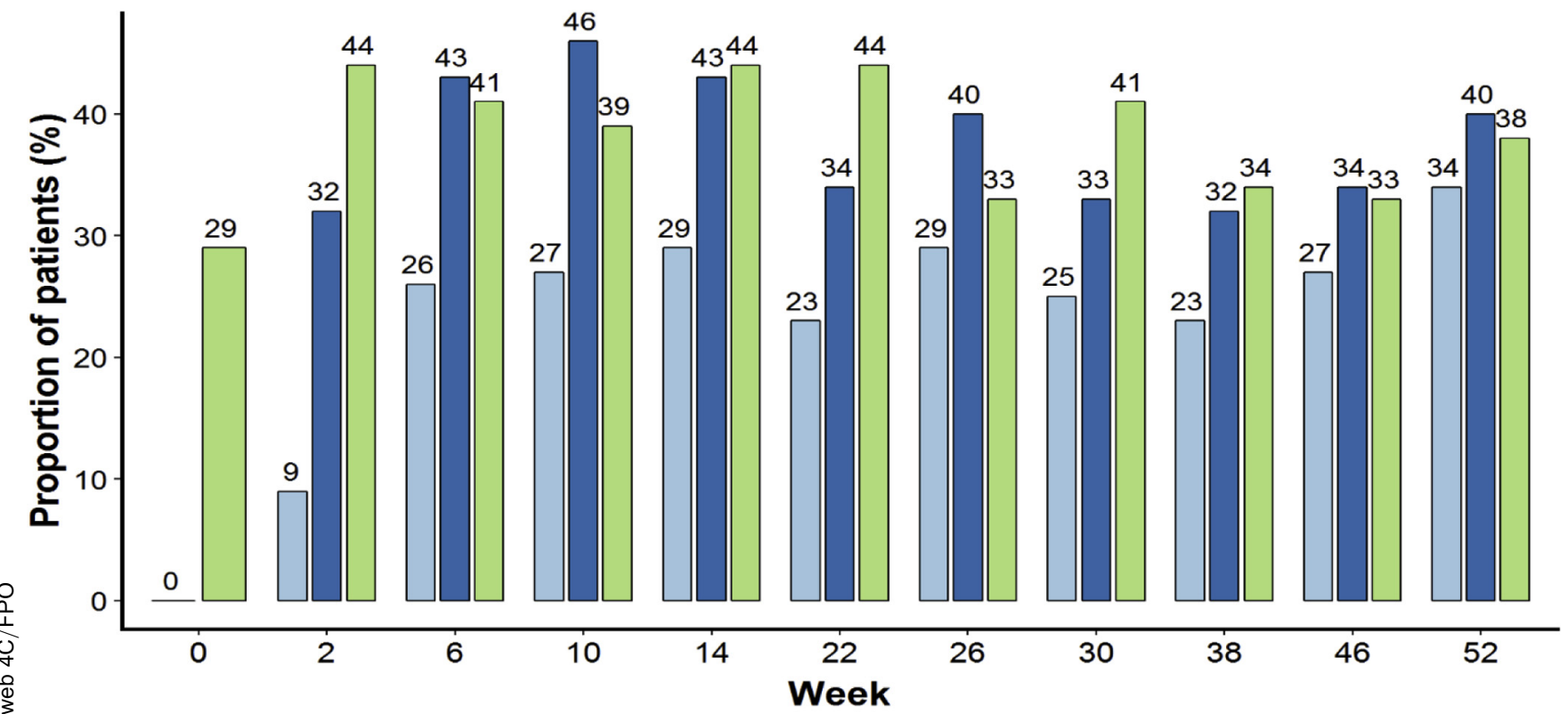

$\square$ CDAI remission $\square$ CDAl response $\square$ CRP normalized

Supplementary Figure 2. CDAI response/remission and CRP concentrations at all time points (ITT).

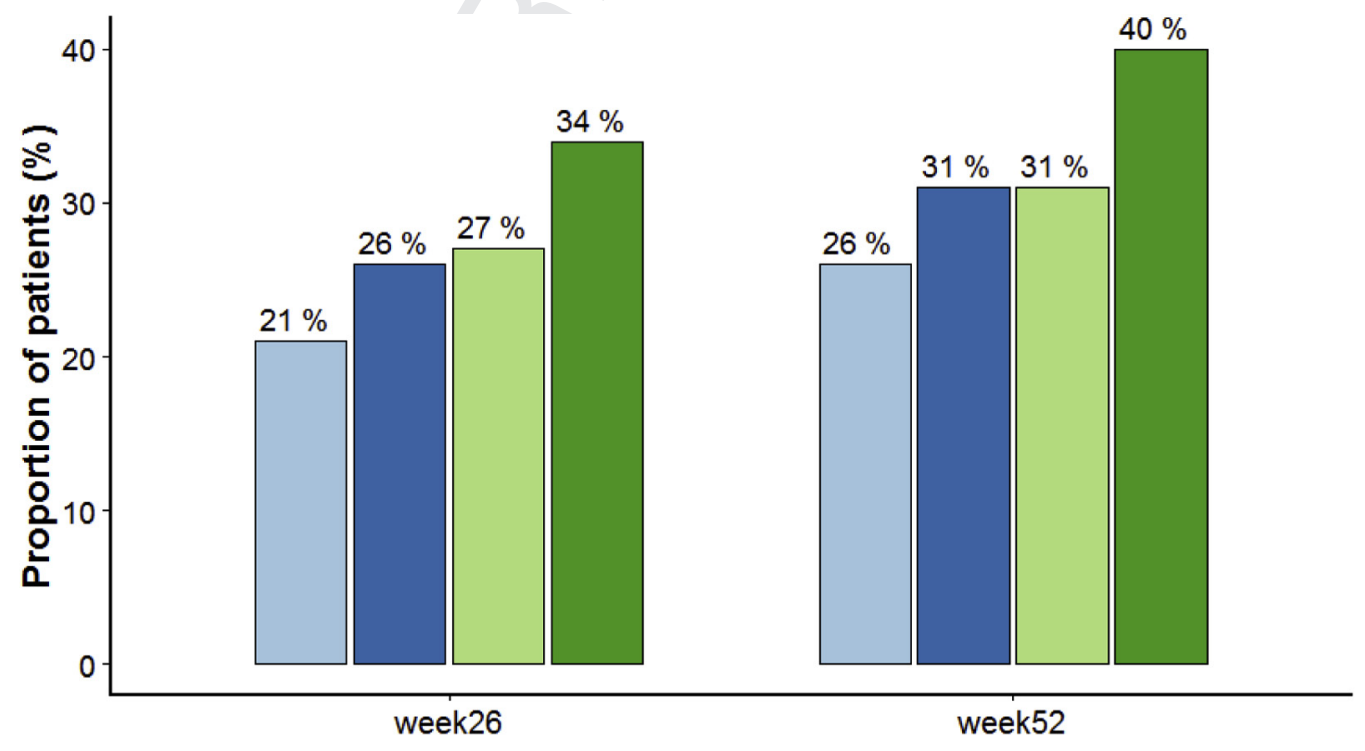

CDAl remission $\square$ CDAl response $\square$ Endoscopic remission $\square$ Endoscopic response

Supplementary Figure 3. Corticosteroid-free clinical remission and endoscopic outcomes in patients receiving additional week 10 infusion.
1381

1382

1383

1384

1385

1386

1387

1388

1389

1390

1391

1392

1393

1394

1395

1396

1397

1398

1399

1400

1401

1402

Q11

1403

1404

1405

1406

1407

1408

1409

1410

1411

1412

1413

1414

1415

1416

1417

1418

1419

1420

1421

1422

1423

1424

1425

1426

1427

1428

1429

1430

1431

1432

1433

1434

1435

1436

1437

1438

1439

1440 


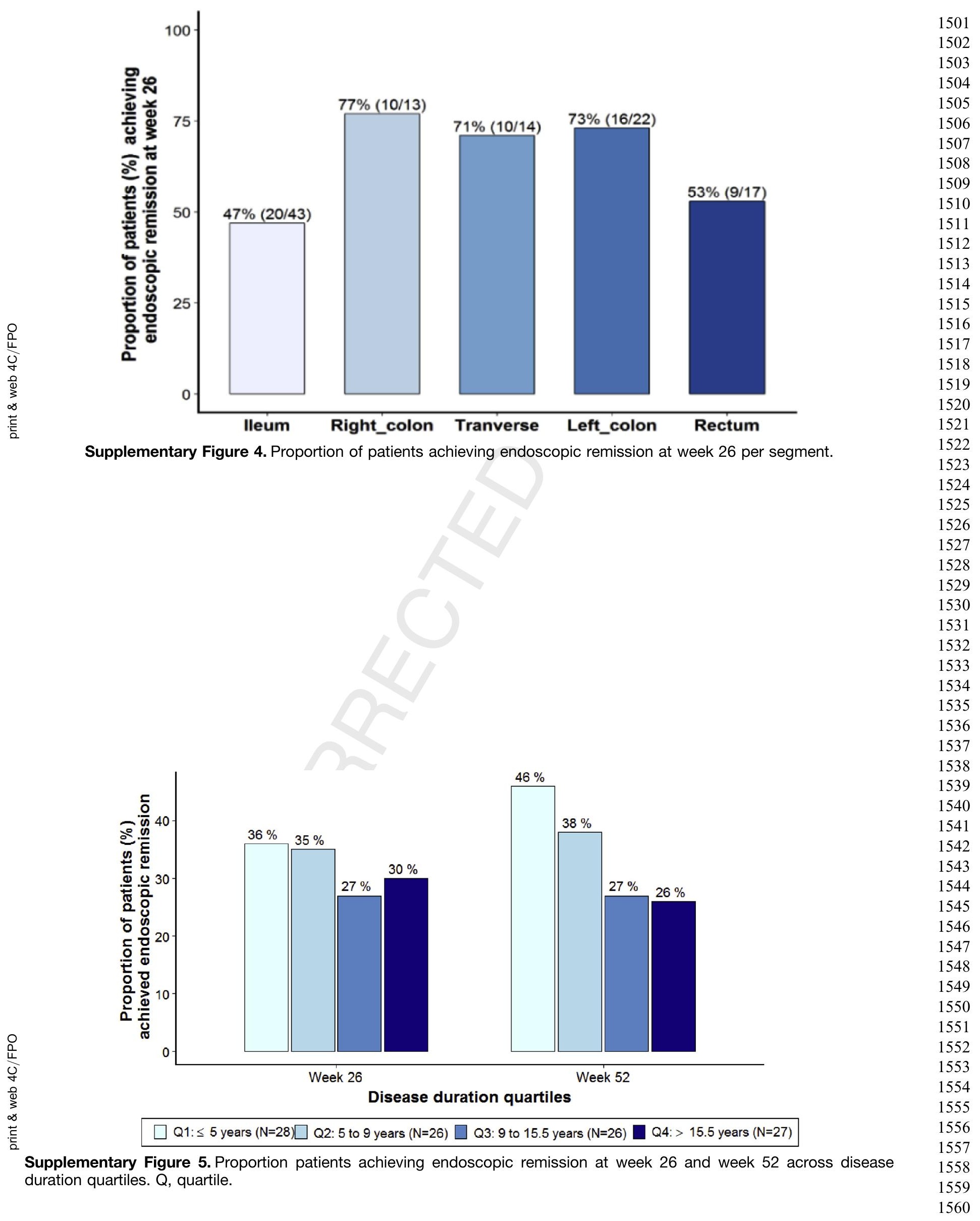




\begin{tabular}{lllll}
\hline Population & $\boldsymbol{n}$ & $\boldsymbol{N}$ & (\%) & $\mathbf{9 5 \%} \mathrm{Cl}$ \\
\hline & & & & \\
All patients & 36 & 110 & 33 & $(24,42)$ \\
& & & & \\
Anti-TNF & & & & \\
Naive & 8 & 13 & 62 & $(32,85)$ \\
Exposed & 28 & 97 & 29 & $(20,39)$ \\
$\begin{array}{l}\text { Prednisone use } \\
\geq 20(\mathrm{mg} / \mathrm{d})\end{array}$ & 2 & 8 & 25 & $(4,64)$ \\
$\begin{array}{l}<20(\mathrm{mg} / \mathrm{d}) \\
\text { Baseline CRP }\end{array}$ & 3 & 12 & 25 & $(7,57)$ \\
$\geq 5$ (mg/L) & 19 & 75 & 25 & $(16,37)$ \\
$<5$ (mg/L) & 15 & 32 & 47 & $(30,65)$
\end{tabular}

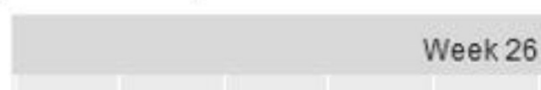$$
<5(\mathrm{mg} / \mathrm{l})(32,0
$$

\section{Week 52}

$$
\text { All patients } \quad 40 \quad 110 \quad 36 \quad(28,46)
$$

\section{Anti-TNF}

$\begin{array}{lllll}\text { Naive } & 8 & 13 & 62 & (32,85) \\ \text { Exposed } & 32 & 97 & 33 & (24,43)\end{array}$

\section{Prednisone use}

$\begin{array}{lllll}\geq 20(\mathrm{mg} / \mathrm{d}) & 2 & 8 & 25 & (4,64) \\ <20(\mathrm{mg} / \mathrm{d}) & 3 & 12 & 25 & (7,57)\end{array}$

\section{Baseline CRP}

$$
\begin{array}{llll}
\geq 5(\mathrm{mg} / \mathrm{L}) & 21 & 75 & 28 \\
<5(\mathrm{mg} / \mathrm{L}) & 16 & 32 & 50
\end{array}
$$

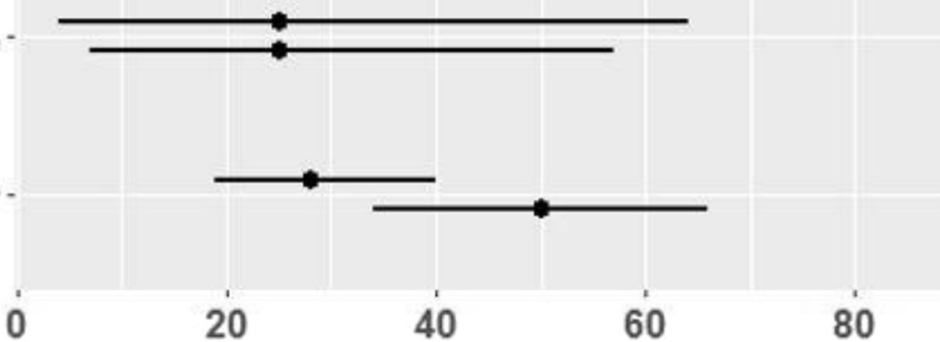

Supplementary Figure 6. Proportion (\%) and 95\% confidence interval of patients achieving endoscopic remission at week 26 (upper panel) and week 52 (lower panel) in the total cohort and stratified by prior anti-TNF use, prednisone use at inclusion, and baseline CRP concentrations.

1622

1623

1624

1625

1626

1627

1628

1629

1630

1631

1632

1633

1634

1635

1636

1637

1638

1639

1640

1641

1642

1643

1644

1645

1646

1647

1648

1649

1650

1651

1652

1653

1654

1655

1656

1657

1658

1659

1660

1661

1662

1663

1664

1665

1666

1667

1668

1669

1670

1671

1672

1673

1674

1675

1676

1677

1678

1679

1680 


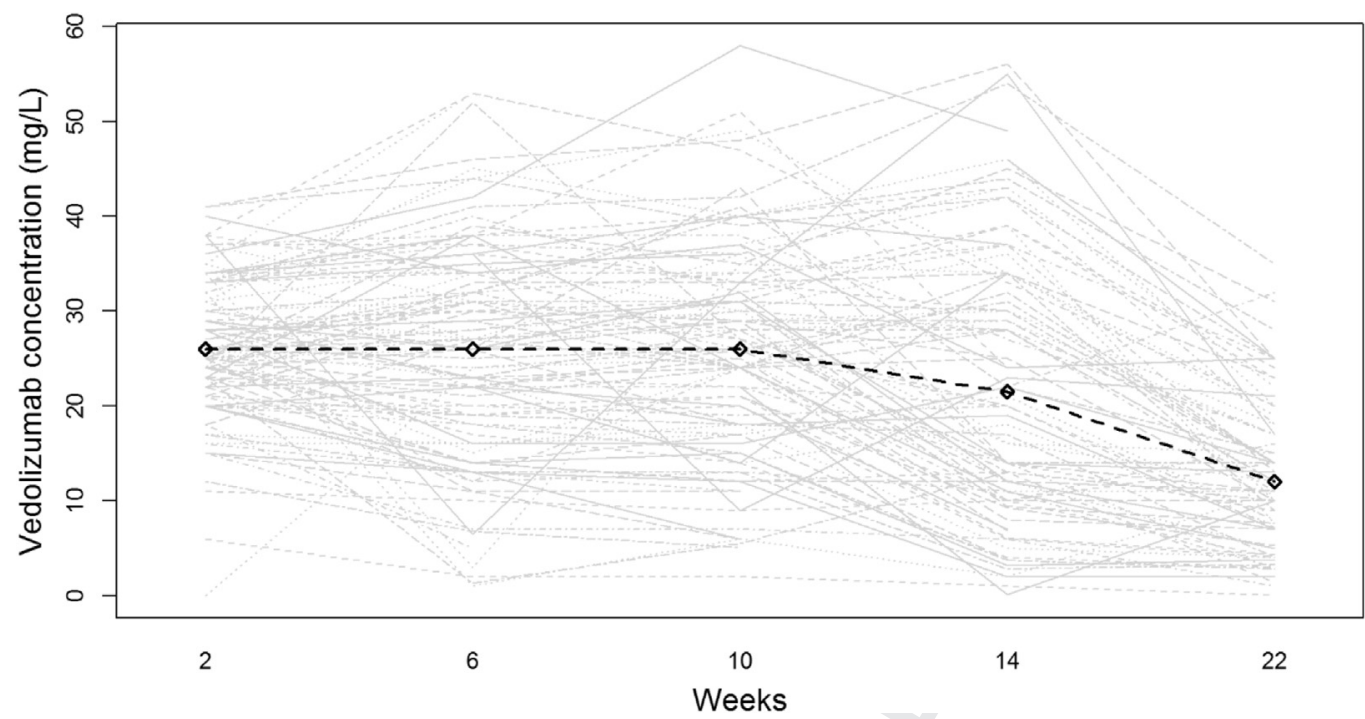

Supplementary Figure 7. VDZ concentrations over time.

Supplementary Table 1.Associations Between Endoscopic and Histological Remission Using the GS and RHI

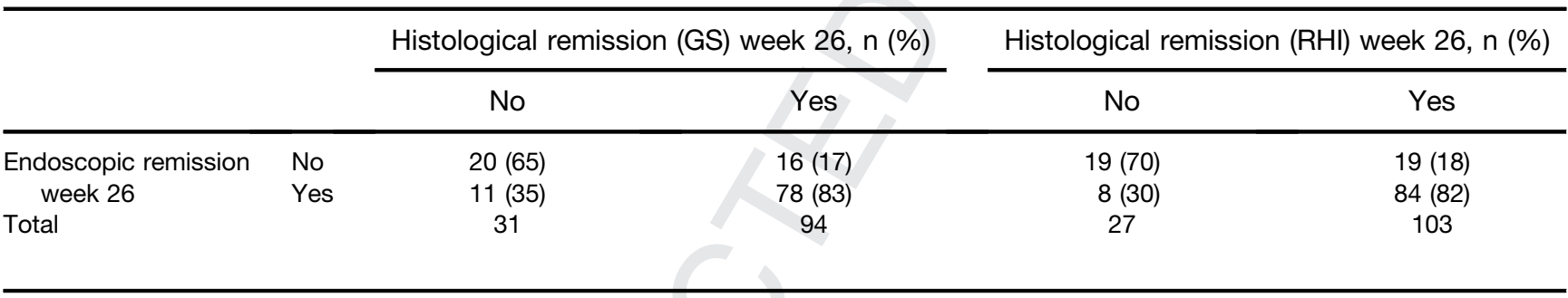

Supplementary Table 2. Correlations Among Clinical, Endoscopic, and Histological Remission at Week 26

\begin{tabular}{|c|c|c|c|c|c|c|c|}
\hline & & \multicolumn{2}{|c|}{$\begin{array}{l}\text { Endoscopic remission } \\
\text { week } 26, \mathrm{n}(\%)\end{array}$} & \multicolumn{2}{|c|}{$\begin{array}{l}\text { Histological remission (RHI) } \\
\text { week } 26, \mathrm{n}(\%)\end{array}$} & \multicolumn{2}{|c|}{$\begin{array}{l}\text { Histological remission (GS) } \\
\text { week } 26, \mathrm{n}(\%)\end{array}$} \\
\hline & & No & Yes & No & Yes & No & Yes \\
\hline \multirow{2}{*}{$\begin{array}{l}\text { Clinical remission } \\
\text { week } 26\end{array}$} & No & $55(74)$ & $23(64)$ & $16(70)$ & $21(50)$ & $17(63)$ & $20(53)$ \\
\hline & Yes & $19(26)$ & $13(36)$ & 7 (30) & $21(50)$ & $10(37)$ & $18(47)$ \\
\hline Total & & 74 & 36 & 23 & 42 & 27 & 38 \\
\hline
\end{tabular}

Supplementary Table 3.ROC Curve Analysis: Different Cutoff Values for Endoscopic Remission at Week 26

\begin{tabular}{lccccccc}
\hline Week & Cutoff & Specificity & Sensitivity & Accuracy & NPV & PPV & $P$ \\
\hline 2 & 23.5 & 0.36 & 0.86 & 0.59 & 0.74 & 0.55 & .06 \\
6 & 25.5 & 0.59 & 0.82 & 0.69 & 0.79 & 0.63 \\
14 & 21.5 & 0.57 & 0.62 & 0.59 & 0.62 & 0.57 \\
22 & 9.9 & 0.54 & 0.91 & 0.71 & 0.87 & .1 \\
& & & & & & & \\
\hline
\end{tabular}

NOTE. $P$ value is related to the area under curve (AUC).

NPV, negative predictive value; PPV, positive predictive value.
1741

1742

1743

1744

1745

1746

1747

1748

1749

1750

1751

1752

1753

1754

1755

1756

1757

1758

1759

1760

1761

1762

1763

1764

1765

1766

1767

1768

1769

1770

1771

1772

1773

1774

1775

1776

1777

1778

1779

1780

1781

1782

1783

1784

1785

1786

1787

1788

1789

1790

1791

1792

1793

1794

1795

1796

1797

1798

1799

1800 
Supplementary Table 4.ROC Curve Analysis: Different Cutoff Values for Endoscopic Response at Week 26

\begin{tabular}{lcccccccc}
\hline Week & Cutoff & Specificity & Sensitivity & Accuracy & NPV & PPV & AUC \\
\hline 2 & 30.5 & 0.38 & 0.72 & 0.57 & 0.54 & 0.58 & 0.55 \\
6 & 19.5 & 0.44 & 0.92 & 0.71 & 0.82 & 0.67 & 0.67 & .2 \\
14 & 6.05 & 0.27 & 1 & 0.66 & 1 & 0.61 & 0.57 & .05 \\
22 & 10.5 & 0.59 & 0.79 & 0.7 & 0.7 & 0.69 & 0.69 & .003 \\
\hline
\end{tabular}

NOTE. $P$ value is related to the area under curve (AUC).

NPV, negative predictive value; PPV, positive predictive value.

\section{Supplementary Table 5.Detectable AVAs}

\begin{tabular}{lccc}
\hline Week & $\begin{array}{c}\text { Nondetectable } \\
\text { AVA (numbers) }\end{array}$ & $\begin{array}{c}\text { Detectable } \\
\text { AVA (numbers) }\end{array}$ & $\begin{array}{c}\text { Detectable } \\
\text { AVA (\%) }\end{array}$ \\
\hline 0 & 102 & 1 & 1 \\
6 & 104 & 4 & 4 \\
10 & 103 & 2 & 2 \\
14 & 94 & 1 & 1 \\
22 & 82 & 1 & 1 \\
38 & 58 & 0 & 0 \\
46 & 57 & 0 & 1 \\
52 & 64 & 1 & \\
& & & \\
\hline
\end{tabular}

Supplementary Table 6. Adverse Events Reported by More

\begin{tabular}{lc}
\multicolumn{2}{c}{ Than 5\% of Patients } \\
\hline \multicolumn{1}{c}{ Adverse event } & $\mathrm{n}(\%)$ \\
\hline Skin lesions $^{\mathrm{a}}$ & $16(15)$ \\
Headache $^{\prime}$ & $13(12)$ \\
Nasopharyngitis & $11(10)$ \\
Arthralgia & $6(5.5)$ \\
Nausea & $6(5.5)$ \\
Any serious adverse event & $\mathrm{b}$ \\
& $2(1.8)$ \\
\hline
\end{tabular}

1861

1862

1863

1864

1865

1866

1867

1868

1869

1870

1871

1872

1873

1874

1875

1876

1877

1878

1879

1880

1881

1882

1883

1884

1885

1886

1887

1888

1889

1890

1891

1892

1893

1894

1895

1896

1897

1898

1899

1900

1901

1902

1903

1904

1905

1906

1907

1908

1909

1910

1911

1912

1913

1914

1915

1916

1917

1918

1919

1920 